\title{
Multicolor lasing prints
}

Van Duong Ta, Shancheng Yang, Yue Wang, Yuan Gao, Tingchao He, Rui Chen, Hilmi Volkan Demir, and Handong Sun

Citation: Appl. Phys. Lett. 107, 221103 (2015);

View online: https://doi.org/10.1063/1.4936628

View Table of Contents: http://aip.scitation.org/toc/apl/107/22

Published by the American Institute of Physics

\section{Articles you may be interested in}

Application of self-assembled hemispherical microlasers as gas sensors Applied Physics Letters 102, 031107 (2013); 10.1063/1.4788751

Tunable whispering gallery modes lasing in dye-doped cholesteric liquid crystal microdroplets Applied Physics Letters 109, 231906 (2016); 10.1063/1.4971973

Resonance energy transfer process in nanogap-based dual-color random lasing Applied Physics Letters 110, 171110 (2017); 10.1063/1.4982161

White light emission with red-green-blue lasing action in a disordered system of nanoparticles Applied Physics Letters 101, 123508 (2012); 10.1063/1.4754286

Active control of emission directionality of semiconductor microdisk lasers Applied Physics Letters 104, 231108 (2014); 10.1063/1.4883637

Tunable single mode lasing from an on-chip optofluidic ring resonator laser Applied Physics Letters 98, 061103 (2011); 10.1063/1.3554362

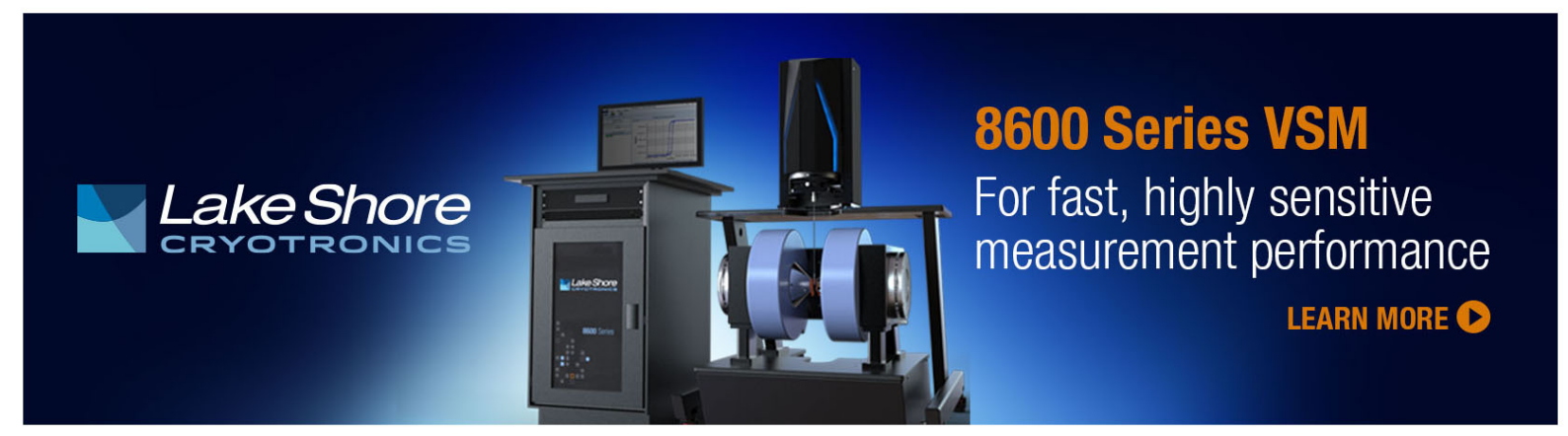




\title{
Multicolor lasing prints
}

\author{
Van Duong Ta, ${ }^{1, a)}$ Shancheng Yang, ${ }^{1, a)}$ Yue Wang, ${ }^{1}$ Yuan Gao, ${ }^{1}$ Tingchao He, ${ }^{1}$ Rui Chen, ${ }^{1}$ \\ Hilmi Volkan Demir, ${ }^{1,2,3,4}$ and Handong Sun ${ }^{1,4, b)}$ \\ ${ }^{1}$ Division of Physics and Applied Physics, School of Physical and Mathematical Sciences, \\ Nanyang Technological University, Singapore 637371, Singapore \\ ${ }^{2}$ School of Electrical and Electronic Engineering, LUMINOUS! Center of Excellence for Semiconductor \\ Lighting and Displays, Nanyang Technological University, Nanyang Avenue, Singapore 639798, Singapore \\ ${ }^{3}$ Department of Electrical and Electronics Engineering and Department of Physics, UNAM-Institute of \\ Materials Science and Nanotechnology, Bilkent University, Bilkent, Ankara TR-06800, Turkey \\ ${ }^{4}$ Centre for Disruptive Photonic Technologies (CDPT), Nanyang Technological University, Singapore 637371, \\ Singapore
}

(Received 10 October 2015; accepted 16 November 2015; published online 30 November 2015)

This work demonstrates mass production of printable multi-color lasing microarrays based on uniform hemispherical microcavities on a distributed Bragg reflector using inkjet technique. By embedding two different organic dyes into these prints, optically pumped whispering gallery mode microlasers with lasing wavelengths in green and red spectral ranges are realized. The spectral linewidth of the lasing modes is found as narrow as $0.11 \mathrm{~nm}$. Interestingly, dual-color lasing emission in the ranges of $515-535 \mathrm{~nm}$ and $585-605 \mathrm{~nm}$ is simultaneously achieved by using two different dyes with certain ratios. Spectroscopic measurements elucidate the energy transfer process from the green dye (donor) to the red one (acceptor) with an energy transfer efficiency up to $80 \%$ in which the nonradiative Förster resonance energy transfer dominates. As such, the acceptor lasing in the presence of donor exhibits a significantly lower $(\sim 2.5$-fold $)$ threshold compared with that of the pure acceptor lasing with the same concentration. (C) 2015 AIP Publishing LLC.

[http://dx.doi.org/10.1063/1.4936628]

Optical microcavities enhancing light-matter interactions by resonant recirculation are building blocks of cavity quantum electrodynamics, light sources, and active filters. ${ }^{1}$ Microresonators with various geometries including FabryPerot, photonic crystal, distributed feedback, and whispering gallery mode (WGM) cavities have demonstrated a wide range of applications. ${ }^{2-5}$ Among these structures, WGM resonators have attracted increasing interests because of not only their promising applications such as ultra-sensitive biosensors, ${ }^{6}$ quantum devices, ${ }^{7}$ low-threshold and high quality (Q) factor microlasers ${ }^{8-10}$ but also their simple fabrication. ${ }^{11-13}$ Indeed, self-assembled droplets can serve as excellent WGM cavities and lasers based on droplet cavities have been realized and used for fast optical switches and gas sensors. ${ }^{14,15}$

Recently, droplet cavities have been greatly benefited from the development of soft matter in optofluidics, ${ }^{16}$ microfluidics, ${ }^{17}$ and inkjet technique. ${ }^{18-20}$ The inkjet method is a cheap and powerful tool for mass production of droplet cavities with highly controlled cavity size and position. ${ }^{19,20}$ Investigation of inkjet method for fabricating high-Q microcavities, like hemispherical resonators, ${ }^{13,21}$ is an essential work because this approach opens opportunities to employ droplets for optical digital devices. ${ }^{22,23}$

Lasers with selectable colors are in high demand for lighting and displays. ${ }^{24,25}$ In particular, multicolor lasers enabled by energy transfer, especially nonradiative Förster

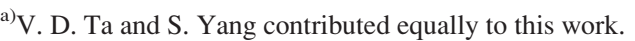

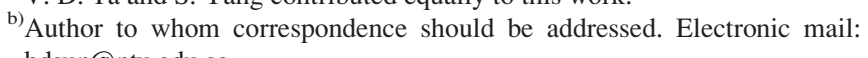
hdsun@ntu.edu.sg
}

resonance energy transfer (FRET), ${ }^{26}$ are very interesting cases because they offer extended lasing wavelengths, high pumping efficiency, and low lasing threshold. ${ }^{27-29}$ In this work, high Q-factor, dual-color lasing from arrays of hemispherical microcavities fabricated by inkjet method is demonstrated.

The laser dyes used were Coumarin 540A (C540A, from Exciton) and Rhodamine $\mathrm{B}$ (RhB, from Sigma-Aldrich). C540A emits green light, which is well absorbed by $\mathrm{RhB}$, and therefore, they make a good pair of donor (D) and acceptor (A), respectively. C540A (15.5 mg, $0.05 \mathrm{mmol})$ and $\mathrm{RhB}$ (24 mg, $0.05 \mathrm{mmol}$ ) were dissolved in dichloromethane (DCM, $0.15 \mathrm{~mL}$, purity $99.76 \%$ ) to make pure donor and acceptor solutions. To achieve multicolor lasing, the two dyes were mixed by adding separately $0.8,1,1.2,1.6,2.4$, 4.9 , and $24 \mathrm{mg} \mathrm{RhB}$ to individual pure C540A solutions, achieving mixtures with various $\mathrm{D}$ :A mole ratios of around $1: 0.03,1: 0.04,1: 0.05,1: 0.07,1: 0.1,1: 0.2$, and 1:1, respectively. Finally, the same amount $(600 \mathrm{mg})$ of Araldite 506 (Sigma-Aldrich) was added into all of the solutions and thus ready for fabricating microcavities.

The hemispherical resonators are deposited on top of a distributed Bragg reflector (DBR) schematically shown in Fig. 1(a). This DBR has a stop-band width of about $140 \mathrm{~nm}$, from 520 to $680 \mathrm{~nm}$, with reflectivity up to $99.9 \%$ at $590 \mathrm{~nm}$ (Fig. 5(c)). The DBR is essential for achieving laser emission as it suppresses the optical loss leaking through the substrate. ${ }^{13}$

To get circular cavity structures, a thin layer of $1 \mathrm{H}, 1 \mathrm{H}, 2 \mathrm{H}, 2 \mathrm{H}$-perfluoroo-ctyltriethoxysilane (a hydrophobic material) was coated on the DBR surface (Fig. 1(b)) before depositing droplets. ${ }^{21}$ Then, hemispherical resonators were 

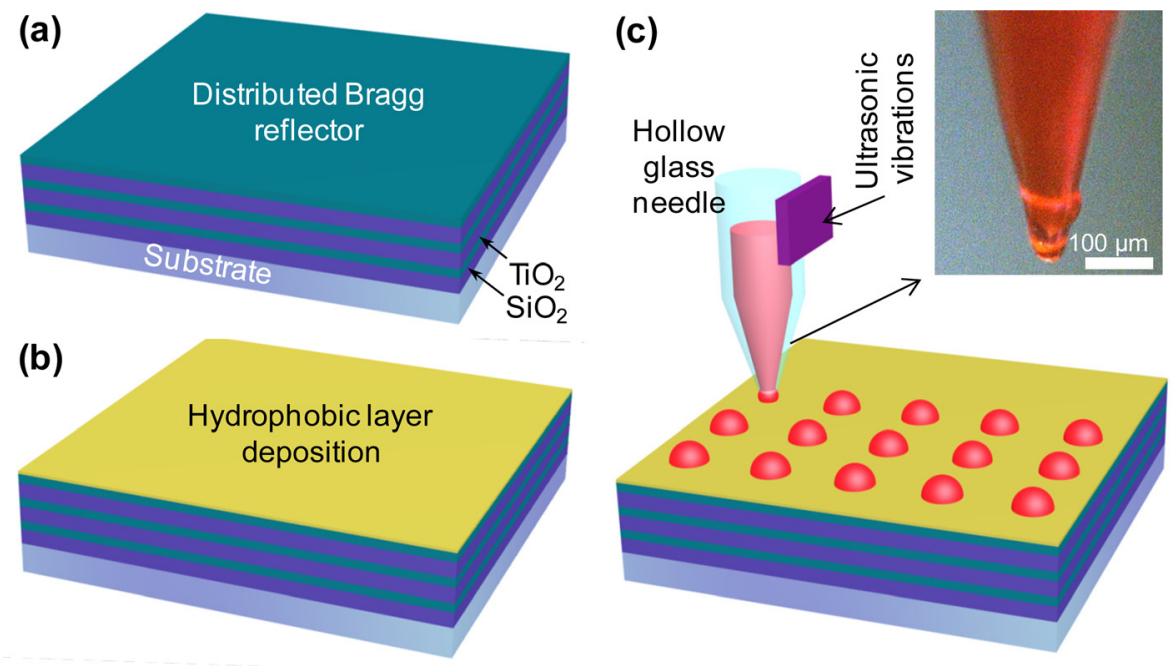

FIG. 1. (a) Schematic of the distributed Bragg reflector (DBR) substrate. (b) The deposition of a hydrophobic thin layer. (c) Fabrication principle of an array of hemispherical resonators on the hydrophobic DBR using a microplotter or inkjet technique. fabricated using a GIX ${ }^{\mathrm{TM}}$ Microplotter ${ }^{\mathrm{TM}}$ II from Sonoplot, INC (Fig. 1(c)). Figures 2(a) and 2(b) show arrays of uniform hemispheres formed using needles whose head diameters are about 30 and $80 \mu \mathrm{m}$, respectively. The cavity sizes rely on the glass needle used, which are about 35 and $85 \mu \mathrm{m}$. To examine the uniformity of the hemispheres, diameters of 173 hemispheres fabricated by the $30 \mu \mathrm{m}$ needle were measured and their size distribution is plotted in Fig. 2(c). It can be seen that about $97 \%$ of the hemispheres have diameters $35 \pm 3 \mu \mathrm{m}$ and approximately $56 \%$ have diameters $35 \pm 1 \mu \mathrm{m}$. Furthermore, the distribution can be fairly fitted by a Gaussian curve.

Hemispheres were investigated using a microphotoluminescence ( $\mu$-PL) setup. It consisted of a tunable $\mathrm{Nd}$ :YAG laser with a wavelength of $420 \mathrm{~nm}$, pulse width of $5-6 \mathrm{~ns}$ and repetition rate of $20 \mathrm{~Hz}$. The laser beam direction was $\sim 45^{\circ}$ normal to the DBR substrate, and the excitation spot has an elliptical shape with the dimension of $\sim 400 \times 500 \mu \mathrm{m}$. Emission from the hemisphere was collected from the top side of the hemispheres by a microscope objective $(50 \times$, numerical aperture 0.42$)$ and recorded by a silicon charge-coupled device (CCD) with a spectral resolution of around $0.06 \mathrm{~nm}$.
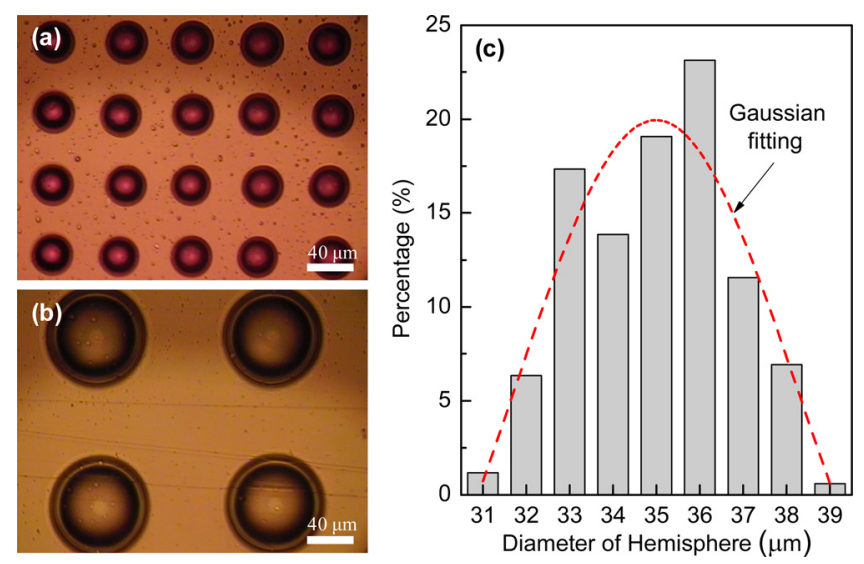

FIG. 2. (a) and (b) Optical images of regular arrays of hemisphere fabricated by a microplotter with 30 and $80 \mu \mathrm{m}$-diameters glass needles, respectively. The structures were doped with $\mathrm{RhB}$ (red) and C504A (green) dyes. (c) Diameter distribution of 173 hemispheres created using the $30 \mu$ m-diameter glass needle.
Laser emissions at green and red wavelengths upon optical pumping were observed from C540A and RhB separately doped hemispheres. Figure 3(a) shows the PL spectra from a $\sim 85 \mu$ m-diameter hemisphere with embedded C540A at pumping energy of about 3.0 and $7.3 \mu \mathrm{J}$. At the low pumping energy, the spectrum was broad, and the intensity of the emission was weak. Therefore, the emission was dominated by spontaneous emission (SE). In contrast, sharp peaks with strong intensity, corresponding to lasing emission, appeared under higher excitation energy. Similarly, even though the absorption of the acceptor is relatively weak at $420 \mathrm{~nm}$, the laser emission at red color (605 to $610 \mathrm{~nm}$ ) was observed from a $\sim 57 \mu$ m-diameter RhB doped hemisphere (Fig. 3(b)). All lasing peaks were well separated with clear free spectral range (FSR) which supports a single cavity loop. Furthermore, due to the high refractive index contrast (0.41), the linewidth of the modes was about $0.11 \mathrm{~nm}$, which is much narrower than $0.3 \mathrm{~nm}^{19}$ and $\sim 1 \mathrm{~nm}^{20}$ reported previously where cavities fabricated by inkjet method from other materials.

The lasing mechanism is ascribed to WGMs and can be characterized by the radial $(r)$ and angular $(m)$ mode number. From the observation, there were no high order modes $(r>1)$ but a fundamental mode $(r=1)$. The mode number $m$ can be simply expressed as $m=\pi n_{\mathrm{e}} D / \lambda_{\mathrm{m}}$, where $\lambda_{\mathrm{m}}$ is the
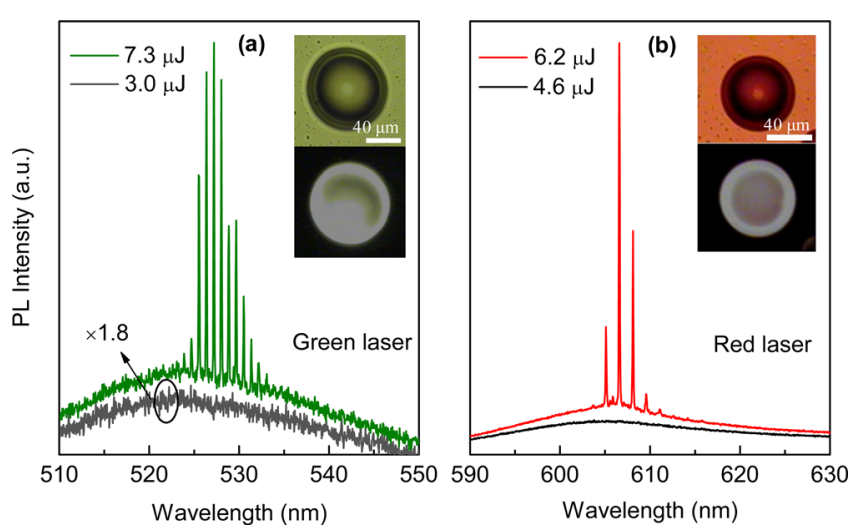

FIG. 3. (a) and (b) Spontaneous and laser emission from C540A and RhB doped cavities under optical pumping. FSR values of the hemispheres are 0.8 and $1.5 \mathrm{~nm}$, respectively. The insets show optical and PL images of the corresponding hemispheres. 
resonant wavelength, $D$ is the diameter, and $n_{\mathrm{e}}$ is the effective refractive index of the hemisphere. Assuming, $n_{\mathrm{e}}=1.41$ and $D=56.69 \mu \mathrm{m}$, then the lasing peaks shown in Fig. 3(b) are well fitted with $m=412-415$. Furthermore, FSR values of the hemispheres shown in Figs. 3(a) and 3(b) are $\sim 0.8$ and $1.5 \mathrm{~nm}$, respectively. They are close to 0.75 and $1.48 \mathrm{~nm}$, predicted by $\Delta \lambda=\lambda_{m}^{2} / \pi n_{e} D$.

To investigate energy transfer process, steady-state PL measurements of pure donor (C540A) and mixture of D-A (C540A-RhB) were performed. Figure 4 shows the normalized PL spectra of pure donor (normalized at the donor emission) and mixture D-A solutions (normalized at the acceptor emission). Stronger emission from the acceptor can be seen while the green emission decreased gradually as the concentration of acceptor increased. The peak intensity of donor emission decreases more than $20 \%$ when concentration of the acceptor is only about $3 \%$ compared with that of the donor. When the mole concentration of donor and acceptor are the same, the peak intensity of the green emission falls sharply to around $7 \%$ of its maximum, which indicates high energy transfer efficiency.

Figure 5 shows that multicolor lasing can be achieved from individual hemispheres with a certain D-A ratio doping. When the concentration of the acceptor is very low ( $\mathrm{D}: \mathrm{A}<1: 0.05$ ), there were lasing modes at the green wavelength and spontaneous orange-red emission from the acceptor (Fig. 5(a)). The gain of the acceptor was not enough for lasing. In contrast, Fig. 5(b) shows that when the D:A ratio is about 1:0.1 or higher, strong acceptor lasing was observed while the emission from the donor was mostly suppressed. Interestingly, by investigating a number of hemispheres with similar sizes and the same concentration of acceptor doping, it is found that threshold of acceptor lasing (D:A is 1:1) was about 2.5 times lower than that of the pure acceptor lasing, which was attributed to the FRET from donor to acceptor (will be discussed later). Multicolor lasing with wavelength of 515-535 nm (green) and 585-605 nm (orange-red) were achieved from both donor and acceptor when D:A is around 1:0.07. For this ratio, proportion of energy from the donor was transferred to the acceptor, contributing to the observation of acceptor lasing, but this energy loss was insufficient to prevent the donor lasing.

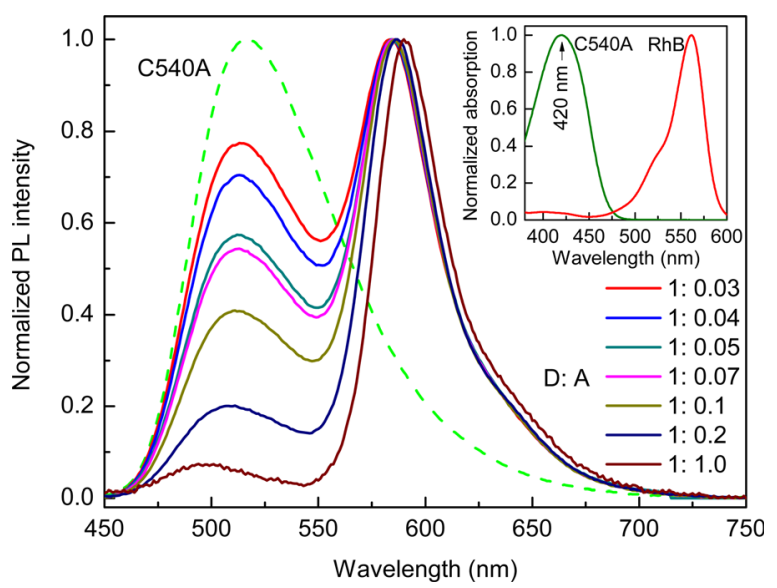

FIG. 4. Normalized PL spectra of pure donor and mixed donor-acceptor dye solutions with various ratios illuminated by Xeon lamp at wavelength of $420 \mathrm{~nm}$. The inset depicts the absorption spectra of the donor and acceptor.
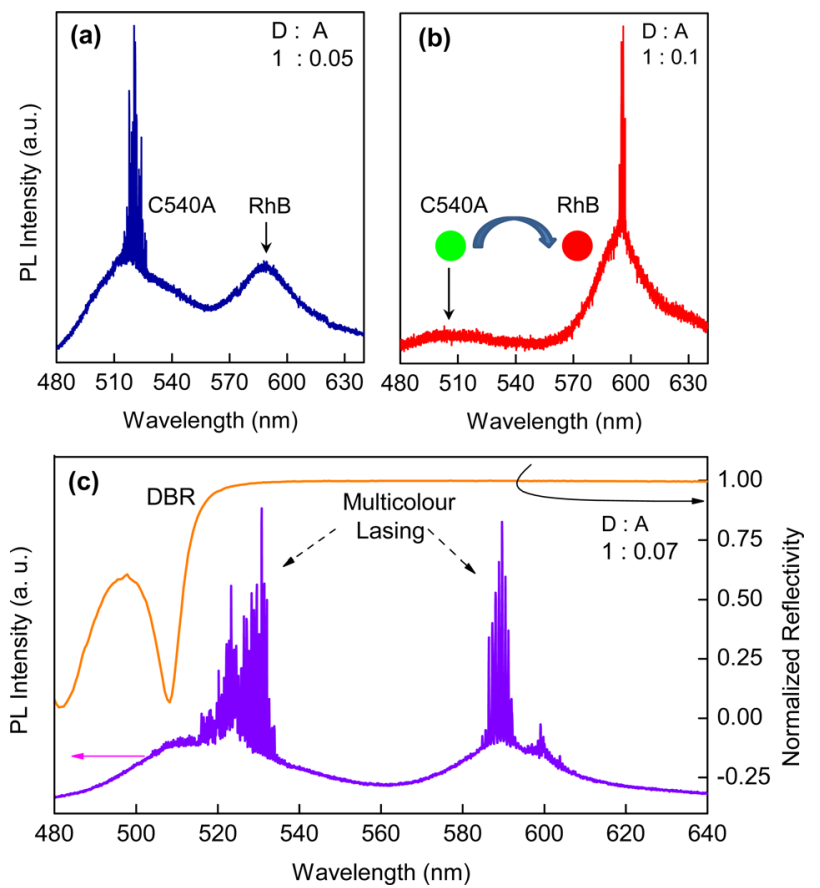

FIG. 5. Lasing spectra from individual hemispheres with various donoracceptor ratios. (a) Donor lasing, (b) FRET-induced acceptor lasing, and (c) dual-colour donor and acceptor laser emission. Stop-band of the DBR used is shown in the top, which completely covers the lasing wavelength range.

The energy transfer process plays an important role in the observation of multicolor lasing. However, energy transfer can be can be either radiative or FRET. ${ }^{30}$ To distinguish the two mechanisms, time resolved-PL measurements were carried for the pure donor and several D-A solutions. These samples were excited by a wavelength-tunable femtosecond laser with a wavelength of $420 \mathrm{~nm}$, pulse duration of $100 \mathrm{fs}$, and a repetition rate of $1 \mathrm{kHz}$. The PL from the solutions were recorded by an Optronics streak camera with a temporal resolution of about 50 ps. Figure 6 plots fluorescence decays of pure donor and mixture of D-A with various ratios, detected at around $520 \mathrm{~nm}$. The curves can be well fitted by

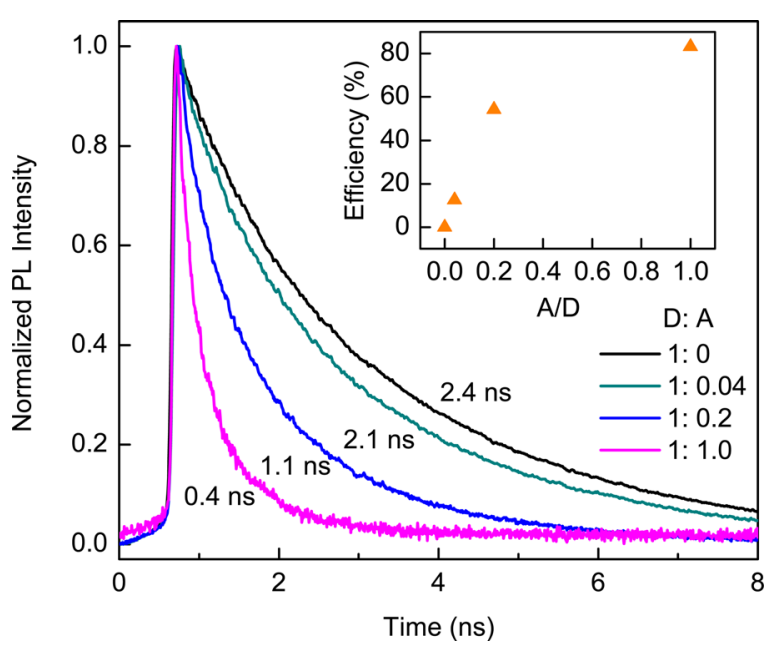

FIG. 6. Fluorescence decay curves of pure donor and donor-acceptor mixtures with various ratios detected at around $520 \mathrm{~nm}$. Extracted lifetimes are shown on each curve. The inset presents energy transfer efficiency as a function of the acceptor:donor ratios. 
an exponential decay function and the lifetime is extracted and given here. It can be seen clearly that the donor lifetime sharply falls with the increasing concentration of the acceptor. Pure donor has the longest lifetime of about $2.4 \mathrm{~ns}$ and decreases to $2.1 \mathrm{~ns}$ when concentration of the acceptor is only $4 \%$ of the donor. When number of acceptors is the same with that of donors, the lifetime of donor shows a significant drop to $0.4 \mathrm{~ns}$.

The efficiency of FRET $\left(\eta_{\mathrm{e}}\right)$ can be estimated from the lifetime as $\eta_{\mathrm{e}}=1-\tau / \tau_{0}$, where $\tau_{0}$ and $\tau$ are lifetimes of pure donor and mixture D-A, respectively. ${ }^{30}$ The inset of Fig. 6 depicts the efficiency of Förster transfer as a function of the A-D ratio, indicating an increase in the energy transfer efficiency from $12.5 \%$ to $83.3 \%$ for A-D of 0.04 and 1 , respectively. This trend is understandable because the D-A separation distance decreases with an increase of acceptor concentration, which leads to the rise of energy transfer rate. As the efficiency of Förster transfer grows higher, FRET plays a more significant role in the acceptor lasing and offers lower lasing thresholds. As a result, FRET-inducted lasing may be potential in high-efficiency lighting and display applications.

In summary, it has been demonstrated that inkjet printing is a highly suitable technique for mass production of high cavity Q-factor hemispherical microcavities (with the resulting Q-factors of the lasing modes $\sim 5 \times 10^{3}$ ). By embedding lasing dye mixtures into these structures, optically pumped lasers with emission at green $(515-535 \mathrm{~nm})$ and orange-red (585-605 $\mathrm{nm}$ ) spectral ranges were achieved. The nonradiative FRET was the dominant effect with energy transfer efficiency up to $80 \%$. Consequently, the acceptor lasing in presence of the donor featured a lower $(\sim 2.5$ fold $)$ threshold compared with the pure acceptor in the absence of donor. It is expected that the emission range of FRETinduced acceptor laser can be further expanded by cascading energy transfer processes through incorporating three or more dyes into the cavities. ${ }^{28}$ White light laser is also possible by adding blue emission, which is significant for lighting and display technology. ${ }^{20,25}$

This work was supported by the Singapore National Research Foundation through the Competitive Research Programme (CRP) under Project No. NRFCRP6-2010-02, the Singapore Ministry of Education through the Academic
Research Fund under Project Nos. MOE 2011-T3-1-005 (Tier 3) and Tier 1- RG92/15, and Merlion under Project No. 2.02.13.

${ }^{1}$ K. J. Vahala, Nature 424, 839 (2003).

${ }^{2}$ J. P. Reithmaier, G. Sek, A. Löffler, C. Hofmann, S. Kuhn, S. Reitzenstein, L. V. Keldysh, V. D. Kulakovskii, T. L. Reinecke, and A. Forchel, Nature 432, 197 (2004).

${ }^{3}$ D. Englund, A. Faraon, I. Fushman, N. Stoltz, P. Petroff, and J. Vuckovic, Nature 450, 857 (2007).

${ }^{4}$ Z. Li, Z. Zhang, A. Scherer, and D. Psaltis, Opt. Express 14, 10494 (2006).

${ }^{5}$ J. Ward and O. Benson, Laser Photonics Rev. 5, 553 (2011).

${ }^{6}$ F. Vollmer and S. Arnold, Nat. Methods 5, 591 (2008).

${ }^{7}$ E. Verhagen, S. Deleglise, S. Weis, A. Schliesser, and T. J. Kippenberg, Nature 482, 63 (2012).

${ }^{8}$ L. He, Ş. K. Özdemir, and L. Yang, Laser Photonics Rev. 7, 60 (2013).

${ }^{9}$ V. D. Ta, R. Chen, L. Ma, Y. J. Ying, and H. D. Sun, Laser Photonics Rev. 7, 133 (2013).

${ }^{10}$ Y. Wang, K. S. Leck, V. D. Ta, R. Chen, V. Nalla, Y. Gao, T. He, H. V. Demir, and H. Sun, Adv. Mater. 27, 169 (2015).

${ }^{11}$ M. Humar, M. Ravnik, S. Pajk, and I. Muševič, Nat. Photonics 3, 595 (2009).

${ }^{12}$ V. D. Ta, R. Chen, and H. D. Sun, Sci. Rep. 3, 1362 (2013).

${ }^{13}$ V. D. Ta, R. Chen, and H. D. Sun, Adv. Mater. 24, OP60 (2012).

${ }^{14}$ S. K. Y. Tang, Z. Li, A. R. Abate, J. J. Agresti, D. A. Weitz, D. Psaltis, and G. M. Whitesides, Lab Chip 9, 2767 (2009).

${ }^{15}$ V. D. Ta, R. Chen, D. M. Nguyen, and H. D. Sun, Appl. Phys. Lett. 102, 031107 (2013).

${ }^{16}$ X. Fan and S.-H. Yun, Nat. Methods 11, 141 (2014).

${ }^{17}$ S.-Y. Teh, R. Lin, L.-H. Hung, and A. P. Lee, Lab Chip 8, 198 (2008).

${ }^{18}$ J. Lee, D. H. Kim, J.-Y. Kim, B. Yoo, J. W. Chung, J.-I. Park, B.-L. Lee, J. Y. Jung, J. S. Park, B. Koo, S. Im, J. W. Kim, B. Song, M.-H. Jung, J. E. Jang, Y. W. Jin, and S.-Y. Lee, Adv. Mater. 25, 5886 (2013).

${ }^{19}$ M. Saito and K. Koyama, Jpn. J. Appl. Phys., Part 1 49, 092501 (2010).

${ }^{20}$ D. J. Gardiner, W. K. Hsiao, S. M. Morris, P. J. W. Hands, T. D. Wilkinson, I. M. Hutchings, and H. J. Coles, Soft Matter 8, 9977 (2012).

${ }^{21}$ J. Haase, S. Shinohara, P. Mundra, G. Risse, V. G. Lyssenko, H. Frob, M. Hentschel, A. Eychmuller, and K. Leo, Appl. Phys. Lett. 97, 211101 (2010).

${ }^{22}$ A. J. C. Kuehne, M. C. Gather, I. A. Eydelnant, S.-H. Yun, D. A. Weitz, and A. R. Wheeler, Lab Chip 11, 3716 (2011).

${ }^{23}$ G. Aubry, Q. Kou, J. Soto-Velasco, C. Wang, S. Meance, J. J. He, and A. M. Haghiri-Gosnet, Appl. Phys. Lett. 98, 111111 (2011).

${ }^{24}$ C. Dang, J. Lee, C. Breen, J. S. Steckel, S. Coe-Sullivan, and A. Nurmikko, Nat. Nanotechnol. 7, 335 (2012).

${ }^{25}$ F. Fan, S. Turkdogan, Z. Liu, D. Shelhammer, and C. Z. Ning, Nat. Nanotechnol. 10, 796 (2015).

${ }^{26}$ T. Forster, Discuss. Faraday Soc. 27,7 (1959).

${ }^{27}$ M. Berggren, A. Dodabalapur, R. E. Slusher, and Z. Bao, Nature 389, 466 (1997).

${ }^{28}$ S. I. Shopova, J. M. Cupps, P. Zhang, E. P. Henderson, S. Lacey, and X. Fan, Opt. Express 15, 12735 (2007).

${ }^{29}$ Y. Sun, S. I. Shopova, C.-S. Wu, S. Arnold, and X. Fan, Proc. Natl. Acad. Sci. U.S.A. 107, 16039 (2010).

${ }^{30}$ R. Chen, V. D. Ta, F. Xiao, Q. Zhang, and H. Sun, Small 9, 1052 (2013). 